\title{
Centralization of Esophageal Cancer Surgery: The Right Thing to Do Is Seldom Easy
}

\author{
Steven DeMeester, MD \\ Department of Cardiothoracic Surgery, Keck School of Medicine, The University of Southern California, Los Angeles, CA
}

In this issue of the Annals of Surgical Oncology Dr. Wouters et al. add further evidence that outcomes for patients undergoing esophagectomy are improved when their surgery is done in a high-volume center. This is an extremely important and timely manuscript for several reasons. First, esophageal cancer, particularly esophageal adenocarcinoma, continues to have the fastest rising incidence in the USA and many Western countries. ${ }^{1}$ Further, the precursor condition for adenocarcinoma of the esophagus, Barrett's esophagus, is also increasing. ${ }^{2}$ Surveillance endoscopy for patients with Barrett's along with the increasing incidence of Barrett's has led to an increase in the number of patients presenting with early-stage esophageal cancer in many centers. The importance of this trend is that the survival for patients with superficial esophageal cancer is completely different from that expected in patients with a locally advanced tumor. Patients with superficial cancers can be cured in nearly all circumstances, and thus very low procedure-related mortality and good long-term quality of life are essential.

A second important issue is that there are now excellent endoscopic alternatives for the therapy of high-grade dysplasia and intramucosal esophageal cancer. Endoscopic resection and ablation has demonstrated efficacy for these conditions, and some centers are beginning to expand the role of endoscopic therapy to include patients with low-risk submucosal tumors. ${ }^{3-5}$ The rationale for endoscopic therapy of these curable lesions is based largely on the morbidity and mortality associated with esophagectomy. Even if it is proven that esophagectomy provides a survival

(C) Society of Surgical Oncology 2009

Published Online: 8 April 2009

S. DeMeester, MD

e-mail: sdemeester@surgery.usc.edu advantage over endoscopic therapy for early esophageal cancer, the ease and widespread availability of endoscopic therapy may make it the preferred approach in many centers. An important point to consider, though, is that, similar to esophagectomy, outcomes with endoscopic therapy may differ between low- and high-volume centers. In fact, it is likely that treatment in a high-volume endoscopic center will be as important for a good outcome with endoscopic therapy as treatment at a high-volume esophageal surgery center is for a good outcome with esophagectomy.

The increasing incidence of esophageal cancer as well as the curability of early-stage tumors mandates that esophagectomy be done in centers with low morbidity and mortality. The results reported by Wouters et al. clearly demonstrate the benefit of centralization of esophagectomies for cancer in The Netherlands, as well as the impact of continuous monitoring of clinical outcomes with feedback to the centers and surgeons involved. The authors indicate that the participating Dutch surgeons agreed upon the scenario of having to refer esophageal cancer patients to centers with a better outcome if their own results proved to be unfavorable. This kind of voluntary outcomes assessment and altruistic referral of patients to other centers would be challenging to implement in the USA for a number of reasons. One is that there is a financial disincentive with this kind of approach for both physicians and hospitals. Referral to another center has an impact well beyond the surgeon, since oncologists, radiation oncologists, hospitalists, and intensivists as well as radiology and laboratory services would all be impacted by the loss of these patients. Second, the USA is geographically much larger than The Netherlands, and determining where the regional centers of excellence would be located and how patients would be transported to these areas would be complex. Third, many patients in the USA prefer to stay near home, at a local community hospital with which they are familiar, rather than go potentially hundreds of miles 
away to an unknown facility, away from family and friends as well as ministers or other members of their support group.

Of course, none of these problems are insurmountable. If malpractice companies stopped providing coverage for esophagectomies by a low-volume surgeon or insurance companies stopped paying for esophageal cancer care in a low-volume or poor-outcome facility this would promptly address the financial disincentive issues for physicians and hospitals. Further, as interested as patients often are in having their health care close to home, they are even more interested in having it paid for by insurance, and so this would also encourage patients to find a way to bring their support group with them to a regional center. This leaves the issue of how to define a high-volume and good-outcome center, and here published results from the experience of Wouters et al. and others, along with data from the National Surgical Quality Improvement Program and other national databases such as that maintained by the Society of Thoracic Surgeons, will begin to set a benchmark that can be used to determine centers of excellence. These centers should be defined, in my opinion, not only on the basis of volume and short-term outcome, but on dedication to cancer follow-up, improving long-term outcome with new and less invasive operations such as the laparoscopic vagal-sparing esophagectomy, and a research focus dedicated to expanding our understanding of the biology of these tumors and developing novel tests and therapies that will improve overall survival even further for patients with this increasing and potentially lethal form of cancer.

\section{REFERENCES}

1. Pohl H, Welch HG. The role of overdiagnosis and reclassification in the marked increase of esophageal adenocarcinoma incidence. J Natl Cancer Inst. 2005;97(2):142-6.

2. van Soest EM, Dieleman JP, Siersema PD, et al. Increasing incidence of Barrett's oesophagus in the general population. Gut. 2005;54(8):1062-6.

3. Ell C, May A, Pech O, et al. Curative endoscopic resection of early esophageal adenocarcinomas (Barrett's cancer)[see comment]. Gastrointest Endosc. 2007;65(1):3-10.

4. Pech O, Behrens A, May A, et al. Long-term results and risk factor analysis for recurrence after curative endoscopic therapy in 349 patients with high-grade intraepithelial neoplasia and mucosal adenocarcinoma in Barrett's oesophagus. Gut. 2008;57(9):1200-6.

5. Manner H, May A, Pech O, et al. Early Barrett's carcinoma with "low-risk" submucosal invasion: long-term results of endoscopic resection with a curative intent. Am J Gastroenterol. 2008; 103(10):2589-97. 